\title{
Effect of tolerance versus chronic immunosuppression protocols on the quality of life of kidney transplant recipients
}

Maria Lucia L. Madariaga, Philip J. Spencer, Kumaran Shanmugarajah, Kerry A. Crisalli, David C. Chang, James F. Markmann, Nahel Elias, A. Benedict Cosimi, David H. Sachs, and Tatsuo Kawai

Center for Transplantation Sciences, Department of Surgery, Massachusetts General Hospital, Boston, Massachusetts, USA.

BACKGROUND. Kidney transplant patients on tolerance protocols avoid the morbidity associated with the use of conventional chronic immunosuppressive regimens. However, the impact of tolerance versus conventional regimens on the quality of life $(\mathrm{QOL})$ of kidney transplant patients is unknown.

METHODS. Five patients who achieved long-term immunosuppression-free renal allograft survival after combined kidney and bone marrow transplantation (tolerant group) were compared with thirty-two comparable kidney transplant recipients on conventional immunosuppression (conventional group). QOL was compared with 16 conventional recipients using the Kidney Disease Quality of Life Short Form 36 (KDQOL SF-36) and the Modified Transplant Symptom Occurrence and Symptom Distress Scale (MTSOSD-59R).

RESULTS. Patients in the tolerant group required significantly less treatment after transplant for hypertension and no medications for diabetes $(P<0.01)$. There was no incidence of diabetes, dyslipidemia, or malignancies in the tolerant group, while these were observed in $12.5 \%, 40.6 \%$, and $11.8 \%$ of the conventional group, respectively. Tolerant patients experienced better overall health $(P<0.01)$ and scored higher on kidney transplant-targeted scales and healthy survey scales than patients in the conventional group according to the KDQOL SF-36 $(P<0.05)$. Tolerant patients were less likely to experience depression, dyspnea, excessive appetite/thirst, flatulence, hearing loss, itching, joint pain, lack of energy, muscle cramps, and lack of libido than conventional patients according to the MTSOSD-59R $(P<0.05)$.

CONCLUSION. Kidney transplant recipients who achieved tolerance experience significantly fewer incidences of complications, improved QOL, and fewer comorbid symptoms compared with patients on conventional immunosuppression. These results support the expanded use of tolerance protocols in kidney transplantation.

Conflict of interest: The authors have declared that no conflict of interest exists.

Submitted: February 10, 2016 Accepted: April 21, 2016 Published: June 2, 2016

Reference information: JCI Insight. 2016;1(8):e87019. doi:10.1172/jci.insight.87019.

\section{Introduction}

Induction of immunologic tolerance is a long-standing goal of organ transplantation, as it achieves acceptance of donor allografts while avoiding the toxicity and costs associated with chronic administration of immunosuppressive drugs. Combined kidney and donor bone marrow transplantation (CKBMT) was first performed in HLA-matched recipients with end-stage renal disease secondary to multiple myeloma in an effort to induce donor chimerism, to treat myeloma, and to achieve successful renal allograft tolerance (1). In 2002, the first successful HLAmismatched kidney transplant under a tolerance-inducing protocol was performed at our institution. Since then, 12 patients have received CKBMT from HLA-mismatched donors. Immunosuppressive therapy was successfully withdrawn, 9-14 months after transplantation, in 8 of these recipients. Three of them resumed immunosuppression 5-7 years after CKBMT due to recurrence of the original kidney disease and/or chronic rejection. The remaining 5 recipients remain off immunosuppression 2.5-14 years after 
Table 1. Characteristics of study participants before and after transplant

\begin{tabular}{|c|c|c|c|c|c|}
\hline Characteristic & Tolerant group $(n=5)$ & $\begin{array}{l}\text { Conventional group } \\
\qquad(n=32)\end{array}$ & $P$ value ${ }^{A}$ & $\begin{array}{l}\text { Conventional group survey } \\
\text { responders }(n=16)\end{array}$ & $P$ value $^{\mathrm{B}}$ \\
\hline \multicolumn{6}{|l|}{ Before transplant } \\
\hline Age at time of transplant $-\mathrm{yr}$ & $30.7 \pm 9.3$ & $35.7 \pm 8.3$ & 0.23 & $35.5 \pm 8.7$ & 0.29 \\
\hline Female sex-no. (\%) & $3(60.0)$ & $13(40.6)$ & 0.42 & $8(50.0)$ & 0.70 \\
\hline $\begin{array}{l}\text { Racial or ethnic minority } \\
\text { group-no. (\%) }\end{array}$ & $0(0)$ & $7(21.8)$ & 0.25 & $4(25)$ & 0.21 \\
\hline Focal glomerulosclerosis & $0(0)$ & $4(12.5)$ & & $1(6.25)$ & \\
\hline Henoch-Schonlein purpura & $0(0)$ & $3(9.4)$ & & $1(6.25)$ & \\
\hline IgA nephropathy & $0(0)$ & $10(31.3)$ & & $7(43.75)$ & \\
\hline Polycystic kidney disease & $0(0)$ & $2(6.3)$ & & $1(6.25)$ & \\
\hline MPGN & $1(20.0)$ & 0 & & 0 & \\
\hline Mean (range) days on HD & $227.3(36-406)$ & $296.1(1-1338)$ & 0.74 & $200(1-398)$ & 0.56 \\
\hline $\begin{array}{l}\text { Medications before transplant-\% } \\
\text { patients }\end{array}$ & & & 0.02 & & \\
\hline Hypertension & 60.0 & 90.6 & 0.06 & 87.5 & 0.17 \\
\hline 1 medication & 20.0 & 28.1 & & 25 & \\
\hline 2 medications & 20.0 & 43.8 & & 50 & \\
\hline$\geq 3$ medications & 20.0 & 18.8 & & 12.5 & \\
\hline Dyslipidemia & 0 & 25 & 0.20 & 25 & 0.21 \\
\hline \multicolumn{6}{|l|}{ After transplant } \\
\hline $\begin{array}{l}\text { Length of hospital stay-mean } \\
\text { (range) d }\end{array}$ & $15.2(8-18)$ & $4.5(3-8)$ & $<0.001$ & $4.7(3-8)$ & $<0.001$ \\
\hline Hypertension & 20 & 78.1 & 0.004 & & 0.03 \\
\hline 1 medication & 20 & 31.3 & & 31.3 & \\
\hline 2 medications & 0 & 40.6 & & 37.5 & \\
\hline$\geq 3$ medications & 0 & 6.3 & & 6.3 & \\
\hline Dyslipidemia & 0 & 40.6 & 0.07 & 31.25 & 0.15 \\
\hline Diabetes requiring insulin & 0 & 12.5 & 0.40 & 0 & - \\
\hline Creatinine (mg/dl)-mean (range) & $1.3(0.9-1.8)$ & $2.41(0.7-8.7)$ & 0.03 & $2.81(1.0-8.7)$ & 0.10 \\
\hline \multicolumn{6}{|c|}{$\begin{array}{l}\text { ESRD, end-stage renal disease; MPGN, membranoproliferative glomerulonephritis; VUR, vesicoureteral reflux; FSCS, focal segmental glomerulosclerosis; } \\
\text { HD, hemodialysis. Racial or ethnic minority group was derived from patient medical records (defined as not of mixed European descent). Length of hospital } \\
\text { stay was measured from day of transplant to day of discharge. Infection was defined as documented infection requiring hospital admission. Creatinine } \\
\text { was measured at time of survey administration. Plus-minus values are mean } \pm \text { SD. }{ }^{A} P \text { value between tolerant group and conventional group. }{ }^{B} P \text { value } \\
\text { between tolerant group and conventional group survey responders. }{ }^{C} \text { Triple: various combinations of calcineurin inhibitor, rapamycin, and belatacept } \\
\text { with antimetabolites (mycophenolate mofetil or azathioprine) and prednisone. }{ }^{D} \text { Dual: various combinations of calcineurin inhibitor or rapamycin with } \\
\text { antimetabolites or prednisone. }\end{array}$} \\
\hline
\end{tabular}

CKBMT (2). Several other centers have also instituted clinical trials of tolerance induction $(3,4)$, and close to 70 patients have undergone or are currently enrolled in tolerance protocols in the United States $(2,5,6)$.

One of the proposed benefits of tolerance induction is that it should limit the deleterious side effects of long-term maintenance immunosuppression. These include not only malignancy and infection, but also 
Table 2. KDQOL SF-36 scores among participants

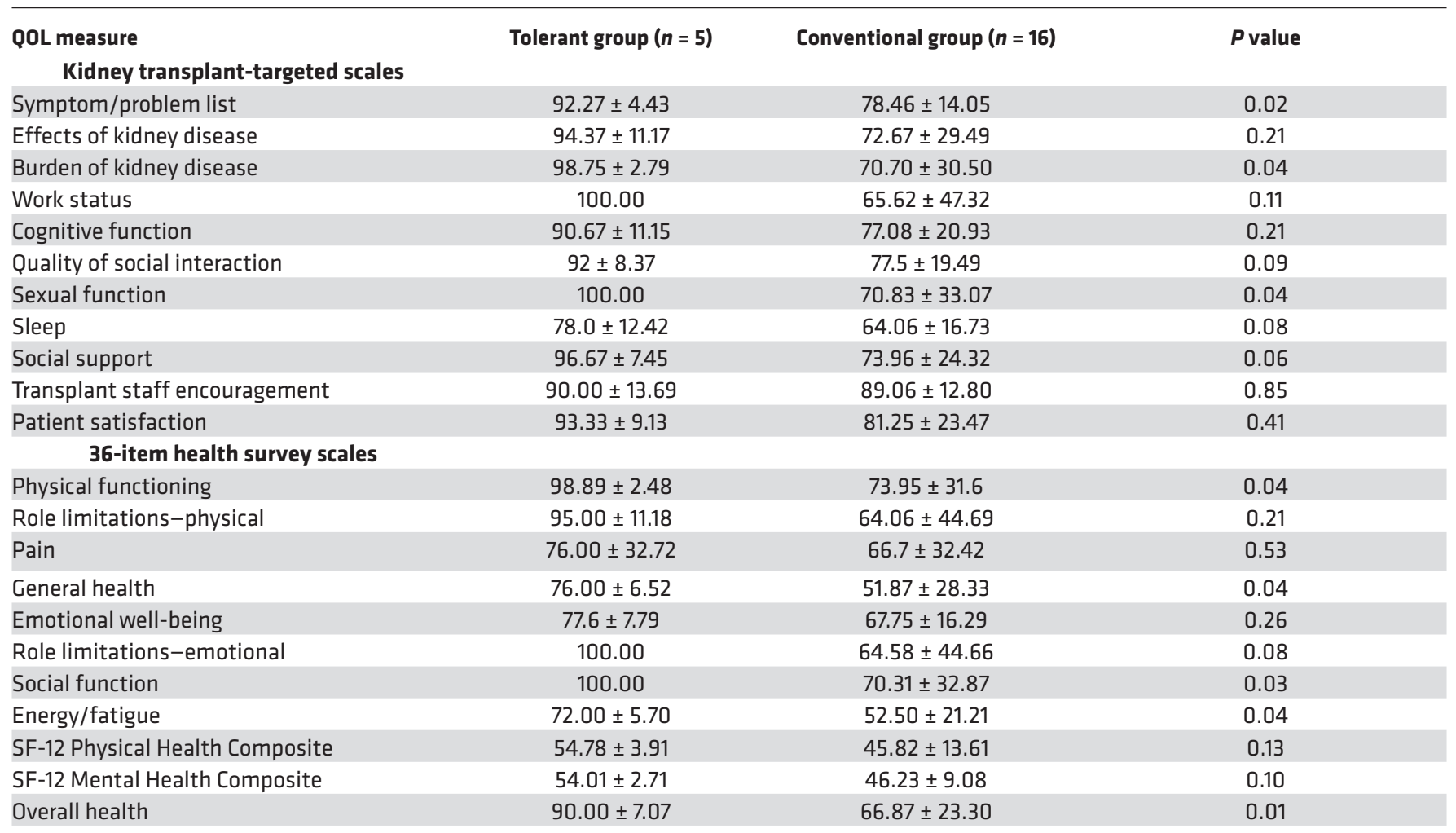

QOL, quality of life. Scoring of Kidney Disease Quality of Life Short Form 36 (KDQOL SF-36) ranges from 0 to 100, with higher scores indicating better outcome. For physical and mental health composite scores, in the general population the mean for each summary scale is 50 points, with a standard deviation of 10 points. Plus-minus values are mean \pm SD. Significance was determined by Wilcoxon rank-sum (Mann-Whitney) test.

drug side effects that may negatively affect the recipient's quality of life (QOL), despite ongoing satisfactory allograft function. Dissatisfaction with the symptom experience of immunosuppressive therapy correlates with poor QOL, triggers medication nonadherence, and can subsequently lead to increased rejection, mortality, and health care costs (7-13).

While it has been shown that kidney transplant recipients experience much better QOL than dialysis patients (14-16), the effect of tolerance protocols on QOL is unknown. Using the Kidney Disease Quality of Life Short Form 36 (KDQOL SF-36) health survey $(17,18)$ and the Modified Transplant Symptom Occurrence and Symptom Distress Scale (MTSOSD-59R) (19), in this study we investigate whether kidney transplant patients who are tolerant of their grafts experience better QOL than a comparable cohort of kidney transplant patients who are maintained on chronic immunosuppression.

\section{Results}

Baseline characteristics. The mean age of the study population at the time of transplant was $35.05 \pm 8.4$ years and $43.5 \%$ were female. There were no significant differences in age, gender, race, end-stage renal disease diagnosis, or need for dialysis after transplant between tolerant and conventional groups (Table 1).

Posttransplant characteristics. In comparison with 32 patients in the conventional group, patients in the tolerant group experienced a significantly longer initial hospital stay (Table 1, mean 15.2 vs. 4.5 days, $P<0.001$ ) and a higher rate of readmissions (because of protocol-mandated serial graft biopsies; mean 5.78 vs. $2, P<0.001)$. In the conventional group, 22 patients were treated with triple immunosuppressive medications with various combinations of calcineurin inhibitor, rapamycin, belatacept with antimetabolites (mycophenolate mofetil or azathioprine), and prednisone, and 10 patients were treated with with dual immunosuppressive medications. In the 5 tolerant patients, no immunosuppressive medication was administered after 9 to 12 months after transplant, and they required significantly less 
Table 3. MTSOSD-59R modified transplant symptom occurrence scale among participants

\begin{tabular}{|c|c|c|c|}
\hline \multicolumn{3}{|c|}{ Symptom occurrence (mean \% occurrence) ${ }^{A}$} & $P$ value \\
\hline & Tolerant $(n=5)$ & Conventional $(n=16)$ & \\
\hline Depressed & 0 & 32.14 & 0.001 \\
\hline Excessive appetite & 0 & 30.36 & 0.006 \\
\hline Flatulence & 0 & 17.86 & 0.04 \\
\hline Hearing loss & 0 & 21.43 & 0.04 \\
\hline Joint pain & 0 & 23.21 & 0.02 \\
\hline Lack of energy & 12.5 & 32.14 & 0.04 \\
\hline Muscle cramps & 0 & 17.86 & 0.02 \\
\hline Reduced interest in sex & 0 & 28.57 & 0.04 \\
\hline Shortness of breath & 0 & 17.86 & 0.02 \\
\hline
\end{tabular}

MTSOSD-59R, Modified Transplant Symptom Occurrence and Symptom Distress Scale. ${ }^{A}$ Symptom occurrence is reported as the average percentage of time that a participant experienced the symptom; symptoms occurred at any point (ranging from never to always).

treatment after transplant for hypertension (Table $1,20 \%$ vs. $78 \%, P=0.004$ ). There was no incidence of new-onset diabetes, dyslipidemia, or malignancies in the tolerant group, while they were observed in $12.5 \%, 40.6 \%$, and $11.8 \%$ in the conventional group, respectively (Table 1 ).

QOL and symptom outcome measures. Five patients (100\%) from the tolerant group and sixteen patients (50\%) from the conventional group completed KDQOL SF-36 and MTSOSD-59R questionnaires. Of the 22 KDQOL SF-36 domains, a significant difference favoring the tolerant group was observed in overall health (Table 2, $P<0.01$ ); additionally, there were possibly significant differences favoring the tolerant group in the symptom/problem list, burden of kidney transplantation, sexual function, physical functioning, general health, social function, and energy/fatigue (Table 2, $P<0.05$ ). Patients in the tolerant group also had better scores compared with patients in the conventional group across all other domains, except for cognitive function and quality of social interaction, though these differences did not achieve statistical significance (Table 2). Of the 59 tested symptoms in the MTSOSD-59R questionnaire, patients in the tolerant group were significantly less likely to experience depression and excessive appetite (Table $3, P<0.01$ ). Patients in the tolerant group also had a trend towards significance in lower likelihood of excessive thirst, flatulence, hearing loss, itching, joint pain, lack of energy, muscle cramps, reduced interest in sex, and shortness of breath (Table $3, P<0.05$ ).

\section{Discussion}

Kidney transplant recipients who achieve tolerance do not require chronic immunosuppressive drugs and therefore are not subject to the significant morbidities associated with their long-term use. In contrast, essentially all conventionally treated renal allograft recipients require life-long medications, not only to suppress rejection, but also to control direct side effects (cardiovascular disease, dyslipidemia, and de novo diabetes) of immunosuppression. Chronic immunosuppression also significantly increases the risk of infection and malignancies (20). While the clinical benefits of achieving tolerance would thus seem clear, the ability of tolerance to improve the QOL of kidney transplant recipients has not been previously reported.

In this study, we review the clinical and QOL outcome measures of kidney transplant recipients successfully weaned from immunosuppression on tolerance protocols compared with a matched cohort of recipients on conventional chronic immunosuppression. We found that tolerant patients had bet- 
ter clinical outcomes than conventional patients. Tolerant patients required less or no medications for hypertension, dyslipidemia, and diabetes (Table 1). In contrast, as documented in numerous reports, the number of conventional patients who required medications for dyslipidemia or diabetes increased (Table 1).

Importantly, we also found that tolerant patients had better QOL outcomes compared with conventional patients. Tolerant patients had better overall health (Table 2, $P<0.01$ ) and tended to have better markers of physical and emotional well-being (Table 2, $P<0.05$ ). Tolerant patients were less likely to experience depression (Table 3, $P<0.01$ ) and had a trend of lower likelihood of excessive appetite/thirst, flatulence, hearing loss, itching, joint pain, lack of energy, muscle cramps, reduced libido, and shortness of breath than conventional patients (Table $3, P<0.05$ ).

Our data, therefore, confirm that achieving tolerance provides clinical and QOL benefits to kidney transplant recipients. QOL is a qualitative measure that can be correlated with morbidity and mortality in kidney transplant recipients (10). Dissatisfaction with the side effect experience of immunosuppressive therapy is related to QOL and can be a trigger for nonadherence $(9,11)$. The incidence of medication nonadherence is frequent among transplant recipients, ranging in the literature from $2 \%$ to $68 \%$ (21). Medication nonadherence in solid organ transplantation is related to poor clinical outcomes, such as rejection, health care costs, and mortality $(7,8,12,13)$. Heart transplant recipients with even minor deviations from the cyclosporine dosing schedule had increased risk for acute rejection episodes (22). Among kidney transplant recipients, nonadherence contributes to up to $20 \%$ of rejection and graft loss (23). Together with data showing that tolerance protocols are more cost-effective (24), our findings support expanding the use of tolerance protocols to more patients requiring kidney transplants as a method of treatment that optimizes long-term benefit.

There are several limitations to our study. First, the sample size of patients is small. It is possible that significant differences in more QOL measures would be identified with increased sample size. Second, our results are biased towards patients who completed their surveys; conventionally treated patients who did not return surveys may have had different reasons for nonparticipation, including worse QOL. Third, due to small sample size, we were unable to perform multivariate analyses to assess predictors of QOL measures.

In conclusion, this study demonstrates that tolerance induction avoids the morbidity of chronic immunosuppression and is associated with better QOL for kidney transplant recipients. A multicenter study to enroll more tolerant patients and comparable nontolerant cohorts would be helpful for confirmation of these results and supporting the benefits of clinical tolerance induction. These results support the development, assessment, and improvement of acceptable tolerance induction regimens for clinical use.

\section{Methods}

Study design. This study was a single-center patient cohort-matched study. Questionnaires (see below) were administered to all patients. Patient electronic medical records were reviewed retrospectively to determine baseline characteristics and those after transplant.

Study participants. A total of 37 patients were selected for inclusion in this study. The tolerant group consisted of 5 patients who successfully achieved long-term $(>13,>6.9,>6.7,>6.4$, and $>2.5$ years $)$ immunosuppression-free allograft survival after HLA haplotype-matched CKBMT. The nonmyeloablative conditioning consisted of cyclophosphamide (or low-dose total body irradiation), anti-CD2 monoclonal antibody (MEDI507) with or without rituximab, and thymic irradiation. After CKBMT, the recipient was treated with calcineurin inhibitor or rapamycin for 9 to 12 months, after which no immunosuppression was administered (2). Three other patients who initially achieved tolerance but needed to resume immunosuppression at the time of this analysis were excluded. The conventional group consisted of 32 consecutive HLA haplotype-matched kidney transplant recipients at Massachusetts General Hospital, matched for age (18-48 years old), cause of kidney disease, and same era of transplantation (2000-2014). All patients in the conventional group received thymoglobulin induction $(1.5 \mathrm{mg} / \mathrm{kg} \times 3)$ before receiving a kidney transplant from a parent or sibling. After transplantation, recipients were treated with either triple or dual immunosuppressive regimens. The median follow-up time for all recipients was 9.3 years (interquartile range, 5.8-11.5 years), with a maximum follow-up time of 13 years. These 32 patients were compared, with 5 tolerant recipients, in the length of the hospital stay, incidence of complications (malignancies, infections), and the medication requirements to treat hypertension, dyslipidemia, and new-onset diabetes. For the QOL study, 16 recipients with conventional immunosuppression who completed the QOL survey were compared with 5 tolerant recipients. 
Questionnaire. QOL was assessed using the KDQOL SF-36, version 1.3, from the Rand Corporation $(17,18)$. The KDQOL questionnaire is a validated QOL instrument that combines the generic SF-36 instrument with a kidney disease-specific instrument. The SF-36 and kidney disease-specific instrument measures domains of functioning and well-being on a 100-point scale, with a higher score reflecting better QOL. Physical and mental health composite summary scores are also calculated; in the general population, the mean for each summary scale is 50 points, with a standard deviation of 10 points. The disease-specific component of the KDQOL was originally targeted towards patients with end-stage renal disease. We replaced references to "kidney disease" with references to "kidney transplant" to adapt the KDQOL for kidney transplant recipients (e.g., "I feel frustrated dealing with my kidney disease" became "I feel frustrated dealing with my kidney transplant.")

The MTSOSD-59R is a subjective appraisal of symptoms associated with side effects (symptom experience) of immunosuppression (19). It includes measurements of symptom occurrence (never to always occurring) and symptom distress (not at all to extremely distressing) on a 5-point rating scale.

Questionnaires were administered to patients by mail. Survey completion and return was encouraged by follow-up phone calls and discussions at routine clinic visits.

Statistics. Pearson's $\chi^{2}$ test was used to compare discrete baseline patient characteristics (e.g., gender, race, diagnosis, medications, HLA mismatch), while the 2-sample Wilcoxon rank-sum (Mann-Whitney) or 2-tailed Student's $t$ test was used to compare continuous variables (e.g., age, time on dialysis, length of stay, QOL scores). The $\alpha$ significance level was set at 0.01 to account for multiple comparisons, given that there were a total of 99 comparisons being made in this study; $P$ values between 0.01 and the typical thresholds of 0.05 were considered "possibly significant" or "trending towards significance." All analyses were performed with the use of STATA version 13.1 (StataCorp LP).

Study approval. The study protocol was approved by the Massachusetts General Hospital institutional review board. Written informed consent was received from participants prior to inclusion in the study.

\section{Author contributions}

MLLM, KS, and TK designed this research study. MLLM, PJS, and KS conducted experiments. MLLM, PJS, KS, and KAC acquired data. MLLM, DCC, and TK analyzed data. MLLM, PJS, KS, KAC, DCC, JFM, NE, ABC, DHS, and TK wrote the manuscript.

\section{Acknowledgments}

M.L.L. Madariaga is an Edward D. Churchill Surgical Research Fellow, Massachusetts General Hospital, and the recipient of a fellowship from the International Society for Heart and Lung Transplantation and a National Research Service Award from the National Heart, Lung, and Blood Institute of the NIH (F32HL117540). P.J. Spencer is an Edward D. Churchill Surgical Research Fellow, Massachusetts General Hospital, and the recipient of a Training Fellowship Grant in Transplantation Biology (T 32 NIH/National Research Service Award 2 T32 AI 7529-16 A1). This work was supported by the Immune Tolerance Network (N01-AI-15416), an international clinical research consortium; the National Institute of Allergy and Infectious Diseases; and the Juvenile Diabetes Research Foundation.

Address correspondence to: Maria Lucia L. Madariaga, GRB-4-425, Massachusetts General Hospital, 55 Fruit St., Boston, Massachusetts 02114, USA. Phone: 516.380.6391; E-mail: mmadariaga@partners.org.

1. Spitzer TR, et al. Combined histocompatibility leukocyte antigen-matched donor bone marrow and renal transplantation for multiple myeloma with end stage renal disease: the induction of allograft tolerance through mixed lymphohematopoietic chimerism. Transplantation. 1999;68(4):480-484

2. Kawai T, et al. Long-term results in recipients of combined HLA-mismatched kidney and bone marrow transplantation without maintenance immunosuppression. Am J Transplant. 2014;14(7):1599-1611.

3. Scandling JD, et al. Tolerance and chimerism after renal and hematopoietic-cell transplantation. N Engl J Med. 2008;358(4):362368.

4. Leventhal J, et al. Chimerism and tolerance without GVHD or engraftment syndrome in HLA-mismatched combined kidney and hematopoietic stem cell transplantation. Sci Transl Med. 2012;4(124):124ra28.

5. Leventhal JR, et al. Immune reconstitution/immunocompetence in recipients of kidney plus hematopoietic stem/facilitating cell transplants. Transplantation. 2015;99(2):288-298.

6. Scandling JD, et al. Chimerism, graft survival, and withdrawal of immunosuppressive drugs in HLA matched and mismatched 
patients after living donor kidney and hematopoietic cell transplantation. Am J Transplant. 2015;15(3):695-704.

7. De Geest S, et al. Incidence, determinants, and consequences of subclinical noncompliance with immunosuppressive therapy in renal transplant recipients. Transplantation. 1995;59(3):340-347.

8. De Geest S, Dobbels F, Fluri C, Paris W, Troosters T. Adherence to the therapeutic regimen in heart, lung, and heart-lung transplant recipients. J Cardiovasc Nurs. 2005;20(5 suppl):S88-S98.

9. Habwe VQ. Posttransplantation quality of life: more than graft function. Am J Kidney Dis. 2006;47(4 suppl 2):S98-S110.

10. Molnar-Varga M, et al. Health-related quality of life and clinical outcomes in kidney transplant recipients. Am J Kidney Dis. 2011;58(3):444-452.

11. Ortega F, et al. Satisfaction and adherence with immunosuppressant treatment in renal transplant patients living with a working graft. J Nephrol. 2013;26(2):297-305.

12. Pinsky BW, Takemoto SK, Lentine KL, Burroughs TE, Schnitzler MA, Salvalaggio PR. Transplant outcomes and economic costs associated with patient noncompliance to immunosuppression. Am J Transplant. 2009;9(11):2597-2606.

13. Vlaminck H, et al. Prospective study on late consequences of subclinical non-compliance with immunosuppressive therapy in renal transplant patients. Am J Transplant. 2004;4(9):1509-1513.

14. Purnell TS, et al. Comparison of life participation activities among adults treated by hemodialysis, peritoneal dialysis, and kidney transplantation: a systematic review. Am J Kidney Dis. 2013;62(5):953-973.

15. Tonelli M, et al. Systematic review: kidney transplantation compared with dialysis in clinically relevant outcomes. Am J Transplant. 2011;11(10):2093-2109.

16. Evans RW, et al. The quality of life of patients with end-stage renal disease. N Engl J Med. 1985;312(9):553-559.

17. Barotfi S, et al. Validation of the Kidney Disease Quality of Life-Short Form questionnaire in kidney transplant patients. $J$ Psychosom Res. 2006;60(5):495-504.

18. Hays RD, Kallich JD, Mapes DL, Coons SJ, Carter WB. Development of the kidney disease quality of life (KDQOL) instrument. Qual Life Res. 1994;3(5):329-338.

19. Dobbels F, Moons P, Abraham I, Larsen CP, Dupont L, De Geest S. Measuring symptom experience of side-effects of immunosuppressive drugs: the Modified Transplant Symptom Occurrence and Distress Scale. Transpl Int. 2008;21(8):764-773.

20. Marcén R. Immunosuppressive drugs in kidney transplantation: impact on patient survival, and incidence of cardiovascular disease, malignancy and infection. Drugs. 2009;69(16):2227-2243.

21. Chisholm MA. Issues of adherence to immunosuppressant therapy after solid-organ transplantation. Drugs. 2002;62(4):567-575

22. De Geest S, et al. Late acute rejection and subclinical noncompliance with cyclosporine therapy in heart transplant recipients. $J$ Heart Lung Transplant. 1998;17(9):854-863.

23. Denhaerynck K, et al. Prevalence, consequences, and determinants of nonadherence in adult renal transplant patients: a literature review. Transpl Int. 2005;18(10):1121-1133.

24. Elias N, Shao S, Gift T, Patel B, Cosimi A, Kawai T. Economic Analysis of Kidney Transplant Tolerance: The Holy Grail Is Less Costly [abstract]. Am J Transplant. 2015;15(supp1 3). http://www.atcmeetingabstracts.com/abstract/economic-analysis-ofkidney-transplant-tolerance-the-holy-grail-is-less-costly/. Accessed May 23, 2016. 